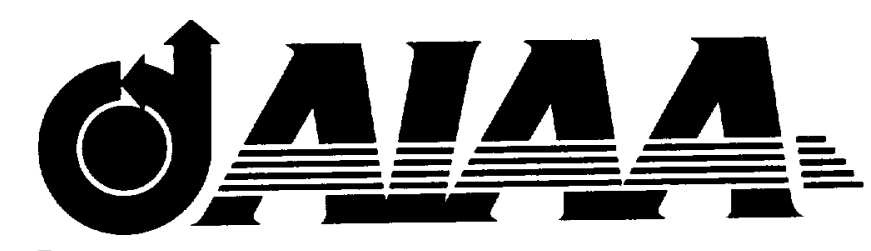

AIAA-2002-0540

Structural Optimization of a Force Balance Using a Computational Experiment Design (Invited)

P.A. Parker, R. DeLoach

NASA Langley Research Center

Hampton, Virginia

\author{
40th AIAA Aerospace Sciences \\ Meeting \& Exhibit \\ 14-17 January 2002 / Reno, Nevada
}



AIAA-2002-0540

\title{
STRUCTURAL OPTIMIZATION OF A FORCE BALANCE USING A COMPUTATIONAL EXPERIMENT DESIGN
}

\author{
P.A. Parker ${ }^{*}$, R. DeLoach ${ }^{*}$ \\ NASA Langley Research Center \\ Hampton. Virginia 23681
}

Abstract

This paper proposes a new approach to force balance structural optimization featuring a computational experiment design. Currently. this multi-dimensional design process requires the designer to perform a simplification by executing parameter studies on a small subset of design variables. This onefactor-at-a-time approach varies a single variable while holding all others at a constant level. Consequently, subtle interactions among the design variables, which can be exploited to achieve the design objectives, are undetected. The proposed method combines Modern Design of Experiments techniques to direct the exploration of the multi-dimensional design space, and a finite element analysis code to generate the experimental data. To efficiently search for an optimum combination of design variables and minimize the computational resources, a sequential design strategy was employed. Experimental results from the optimization of a non-traditional force balance measurement section are presented. An approach to overcome the unique problems associated with the simultaneous optimization of multiple response criteria is described. A quantitative single-point design procedure that reflects the designer's subjective impression of the relative importance of various design objectives, and a graphical multi-response optimization procedure that provides further insights into available tradeoffs among competing design objectives are illustrated. The proposed method enhances the intuition and experience of the designer by providing new perspectives on the relationships between the design variables and the competing design objectives providing a systematic foundation for advancements in structural design.

\footnotetext{
* Research Scientist

- Senior Research Scientist

Copyright $(2001$ by the American Institute of Aeronautics and Astronautics, Inc. No copyright is asserted in the United States under Title 17. U. S. Code. The U. S. Government has a royalty-free license to exercise all rights under the copyright claimed herein for Governmental Purposes. All other rights are reserved by the copyright owner.
}

Introduction

Direct force and moment measurement of aerodynamic loads is fundamental to wind tunnel testing. Typically these measurements are made with an instrument known as a force balance, which is internally mounted in a scaled wind tunnel model (see Figure 1). The force balance is a complex structural spring element. which provides high-precision measurements of the aerodynamic loads exerted on the wind tunnel model, by measuring strain within its flexural elements. This measured strain is then converted into an electrical signal. Electrically measured strain as a function of an externally applied load forms the basic concept of force balance measurements.

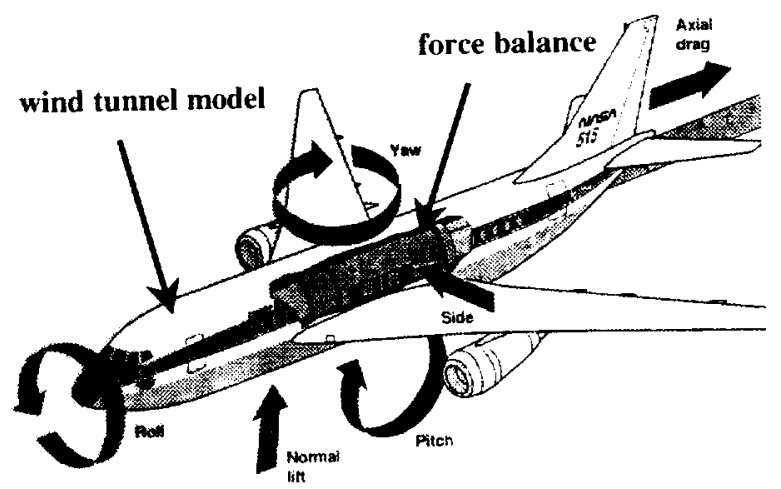

Figure 1. Internally mounted force balance.

The balance flexural elements are designed such that the magnitude of the strain, at strategic locations, is the same for the individual application of each component of load, even though the magnitudes of the components are not equal. Therefore, the flexural element stiffness must be simultaneously tuned with respect to the component loads in six degrees of freedom. An adequate balance design is achieved through a combination of the structural design of multiple flexural elements, and the strategic location of the strain gages. Auxiliary factors must also be considered by the balance design engineer including the 
complexity of fabrication, which is directly proportional to both the production time and cost of the force transducer. Ultimately, the physical design is constrained by the available space within the wind tunnel model.

Currently, structural optimization of the force balance relies heavily on the experience and intuition of the balance designer. Software based analysis and simulation tools have been introduced in recent years to aid in this process', but the decisions regarding design variables are solely driven by the balance designer. These design variables (independent variables) include the measuring section dimensions, shape, location, and strain gage placement.

The quality of the design is evaluated based on multiple competing response metrics (dependent variables) such as: achieving an acceptable sensitivity of the measurements, maximizing the stiffness, minimizing the combined stress intensity, and minimizing the fabrication complexity. There is typically on the order of 20 independent variables, and 10 dependent variables. The goal of this multidimensional design process is to find an optimum combination of the design variables that creates the best overall performance of the response metrics within the constraints.

It is very difficult to reconcile the effects of such a large number of design variables on so many competing response metrics by conventional one-factor-at-a-time (OFAT) methods. In practice the balance engineer typically uses his intuition to effect a simplification of the problem. This simplification is often achieved by considering only a relatively small subset of the design variables, and focusing primarily on a few of the response metrics believed to be most critical. Even in this case. OFAT methods make it difficult to resolve potentially important interactions among the design variables.

Past research in this area has identified the structural optimization of a force balance as an emerging area of force balance development. Zhai ${ }^{2}$ and others identified the need for optimizing the structure of the balance to achieve the desired high sensitivity, low interactions. and high stiffness. An empirical algorithm, which simulates biological growth, was combined with classical finite element analysis to develop new nexure shapes. Zhai ${ }^{3}$ and Parker ${ }^{4.5}$ highlight the benefits of a stiffer balance, namely that it will raise the natural frequency of the model, balance, sting system thereby reducing undesirable model vibration. The desirability of high sensitivity, or flexibility, and high stiffness emphasizes the competing criteria involved in the structural optimization. Parametric studies of beam dintensions were performed using an OFAT approach to develop optimum flexural geometry. ${ }^{3}$ An important finding of this effort was that only minor improvements are possible without investigating new structures and shapes.

Iterative gradient-based algorithms were applied in Reference 6. In this effort, Hou and Twu identified the ability to automate the design of balance flexural sections. The main goal of this work was to maximize the sensitivity, while minimizing the stress intensity. Once again. the competing nature of the criteria is highlighted by the desire to have high stress levels in the local vicinity of the strain gage, while minimizing the stress globally over the flexural element.

\section{Approach}

This paper proposes the use of a computational experimental design using Modern Design of Experiments (MDOE) techniques combined with a state-of-the-art finite-element analysis code to generate the experimental data. The goal of the proposed method is to efficiently move through the multidimensional design space incorporating the competing criteria for the responses.

The proposed technique augments the intuition of the designer by providing graphical insight to the relationship between the design variables and the response metrics. This insight differs from other structural optimization methods that could be used such as: genetic algorithms, neural networks, and gradient search techniques. These methods search the design space to find a maximum response location, but many times do not provide vital information on why a particular path through the design space was chosen. In contrast, the current effort builds mathematical models of a portion of the design space providing an representation of its landscape. These models provide the balance designer with the relative influence of the design variables and valuable information about the subtle interactions among them. Other optimization methods commonly provide an answer without an explanation, while the use of experimental design techniques offers both an answer, an explanation, and new insights to the balance design engineer.

A sequential design strategy was employed in order to minimize the required number of computational runs. thereby increasing computational efficiency. This sequential strategy relies on lower order mathematical models initially, that account for the main effects of the design variables and the interactions among them. These simple, initial models serve as a relatively lowcost means of identifying efficient directions for further experimentation. This strategy then involves an increase in the order of fitted models in the more 
interesting regions of response extrema (maximum or minimum responses.) Once an adequate model has been generated, an optimization is performed to determine the combination of design variables that maximizes a weighted desirability function that combines multiple response metrics.

\section{Experimental Results}

This section details an example of the optimization of a force balance measurement section using the proposed technique. A definition of the design and response variables is provided followed by a discussion of the experimental design and analysis. The final process of using the derived mathematical model to perform an optimization is presented.

\section{Geometry Description}

A typical six-component force balance used at NASA Langley Research Center consists of three measurement sections as shown in figure 2. These measurement sections are the areas of concentration for the structural design. The center section is designed to measure axial force, or drag. The two sections on either side of the axial section are designed to measure the other five components of load and are referred to as the cage sections. The term cage section comes from the classical geometry of multiple rectangular flexures used in this section creating a cage type structure. A parallel system of rectangular flexures is used because it is relatively easy to analyze, but does not necessarily represent optimum geometry. In fact the sharp corners of the rectangular flexures create locations of stress concentration. especially due to torsional loads. On either side of the cage section are solid elements of the balance structure, which are referred to as bulkheads. These bulkheads are considered rigid compared to the flexural elements of the measurement sections. The balance moment center, which is a reference location for the torque components, is typically defined to be at the center of the axial measurement section, and therefore the cage sections are symmetric about the balance moment center.

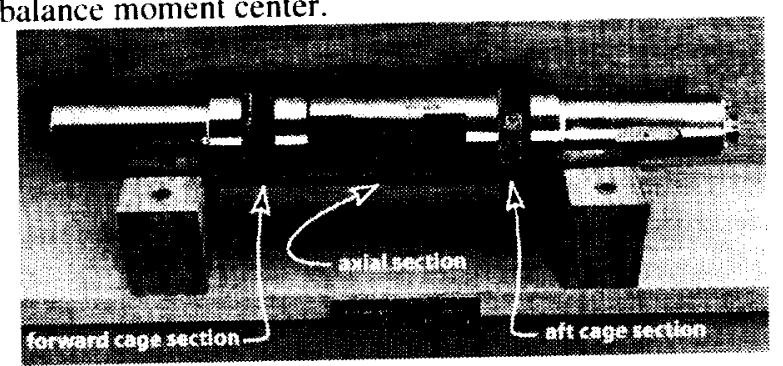

Figure 2. Typical force balance measurement sections.
An advanced geometry cage section was selected for optimization. As previously mentioned, minimizing the number of flexures that comprise the cage section is desirable from a manufacturing point of view; therefore a single flexural element was considered. It is believed that a hollow elliptical cross section would be optimum. For this experiment, the elliptical shape has been approximated by an octagon with a hole in the center. This approximation is due to manufacturing difficulty of an elliptical beam. The design variables control the volume, shape, contour, and location of the cage section.

A generic octagonal cross section is shown in figure 3a. The four linear dimensions of the cross section. the diameter of the center hole, and the two radii that provide a smooth transition between the sides of the octagon represent seven of the design variables. A side view of the flexural element is provided in figure 3b. In this figure, the location of the cage section with respect to the balance moment center, the length of the flexure, and the symmetrical radii at the ends of the flexure represent three additional design variables. A summary of these ten design variables is provided in Table I.

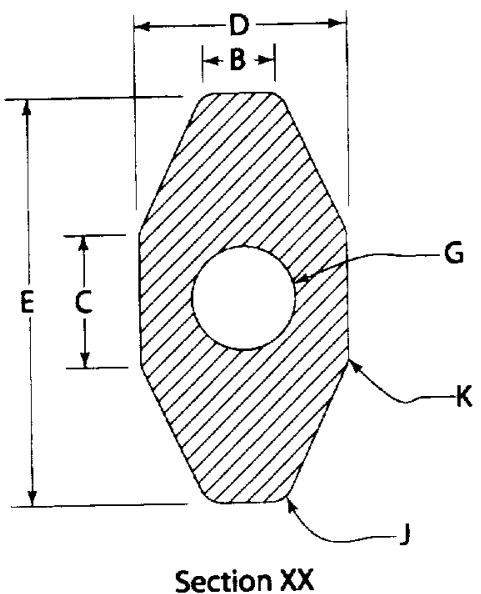

(a) Octagonal cross section.

Figure 3. Design variables.

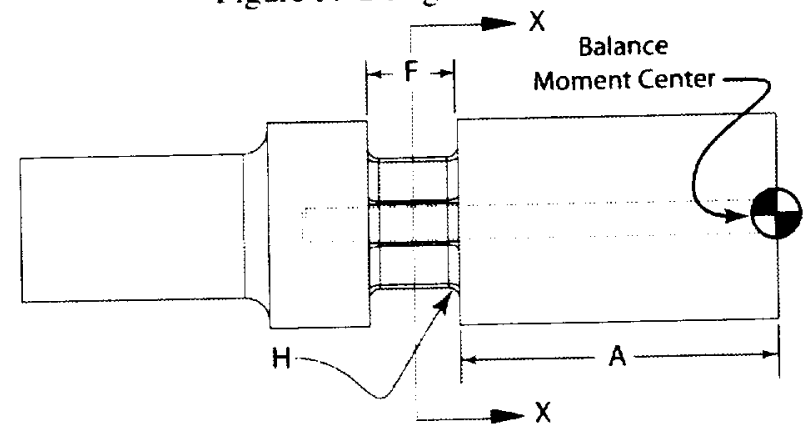

(b) Side view of the flexure.

Figure 3. concluded. 
Table I. Summary of the design variables

\begin{tabular}{|l|l|}
\hline $\begin{array}{l}\text { Design } \\
\text { Variable }\end{array}$ & Description \\
\hline A & location of cage section \\
\hline B & minor width \\
\hline C & minor height \\
\hline D & overall width \\
\hline E & overall height \\
\hline F & length of tlexure \\
\hline G & hole diameter \\
\hline H & radii at ends of flexure \\
\hline J & longitudinal radii along top of flexure \\
\hline K & longitudinal radii along side of flexure \\
\hline
\end{tabular}

The response variables determine the quality of the structural design of the force balance. As previously mentioned. the goal is to maximize the sensitivity while minimizing the structural deflection and overall stress intensity. The four measurement sensitivities chosen for this experiment were normal force, pitching moment. yawing moment, and side force as shown in figure 1. The strain gages used to measure these components are located at the longitudinal centerline of the flexure on the surfaces with the maximum distance from the neutral axis with respect to the component of load. The strain gage locations are shown in figure 4 . The balance utilizes an electrical network of four strain gages configured in a Wheatstone bridge arrangement. In this experiment a single location was used to determine the sensitivity due to the symmetric placement of the four gages in the bridge. Furthermore, this component measurement strategy uses the same gage to measure normal force as well as pitching moment. Normal force is measured by adding the signals from the two symmetric cage sections (forward and aft of the balance moment center) and pitching moment is measured by subtracting the signals. The yawing moment and side force are measured in a similar arrangement.

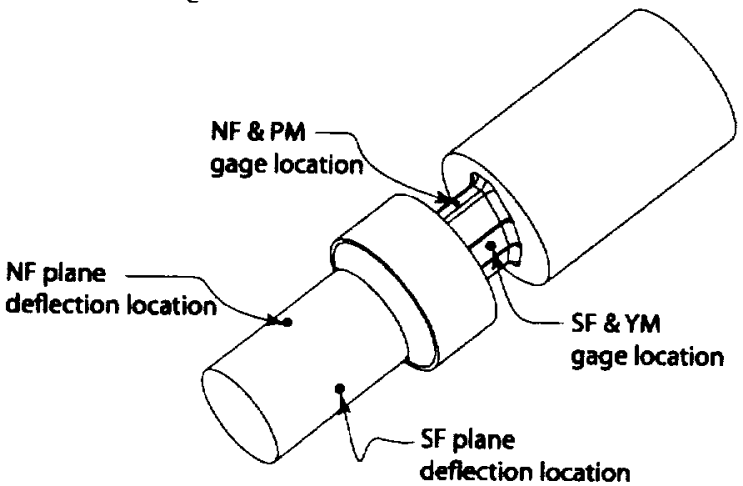

The rolling moment component, which would typically be measured in the cage section, was not included in this initial investigation for the purpose of simplification. The location of the strain gage for rolling moment could be anywhere on the flexure depending on the cross section. This would increase the number of design variables to at least thirteen, and add considerable complexity to the automated aspects of the finite element solution and results interpretation. Including rolling moment in future work would provide a significant advantage over current methods.

Additional response variables were included that were used to minimize the structural deflection and the stress intensity. The structural deflection is measured at a location forward of the cage section as shown in figure 4 . There are two response variables for the deflection, one in the normal force plane and one in the side force plane. It is desirable to minimize both of these simultaneously. The stress intensity was calculated by superposition of the maximum von Mises stress due to the application of the four components of load under study (normal, pitch, yaw, and side). The location of the maximum stress was not considered for each flexure configuration, but intuitively it will be located at the transition between the cage section and the bulkheads. A summary of the response variables is provided in Table II.

Table II. Summary of the response variables

\begin{tabular}{|l|l|}
\hline $\begin{array}{l}\text { Response } \\
\text { Variable }\end{array}$ & Description \\
\hline NF_out & normal force sensitivity \\
\hline PM_out & pitching moment sensitivity \\
\hline YM_out & yawing moment sensitivity \\
\hline SF_out & side force sensitivity \\
\hline zdisp & displacement in the normal plane \\
\hline ydisp & displacement in the side plane \\
\hline max_stress & maximum Von-mises stress \\
\hline
\end{tabular}

To summarize the experiment parameters, there are ten design variables (independent variables) and seven response variables (dependent variables). We want to obtain a mathematical model that cnables the prediction of the values for the response variables from a given combination of the design variables. With this model we are able to search through the design space to find an optimum combination of design variables to achieve the combined response objectives. The experimental data required to generate the model was obtained using a finite element analysis package described in the next section.

Figure 4. Response measurement locations. 
Solid Geometry Modeling and Finite Element Analysis Description

A parametric solid model was used to generate the geometry for the finite element analyses. The representation of the force balance was simplified to minimize computational resources. The force balance was longitudinally cut in half at the balance moment center. All of the features except those necessary to model the flexure boundary conditions, apply the loads. and properly constrain the system were removed. The simplified geometry is shown in figure 5. A table of parameters that were specified by the experimental design controlled the geometry. Each combination of design variables was verified to ensure that it did not violate the geometrical constraints. This process of verification will be described in more detail in the next section describing the experimental design.

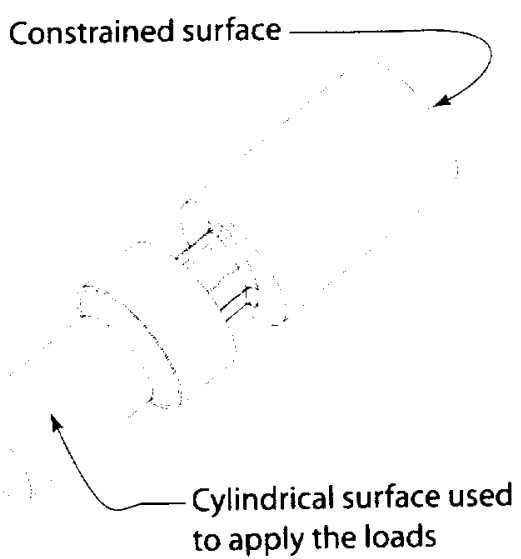

Figure 5. Parametric solid geometry.

The finite element analysis (FEA) package used for this experiment is hased on p-element technology. Pelements rely on a relatively coarse discretization of the geometry using solid tetrahedral elements. Differing from classical finite element analysis codes, the transfer functions through these elements are high-order polynomial functions. This type of FEA has the desirable property of self-refining its ability to represent steep stress intensity gradients found in geometry transitions. In a classical FEA system the analyst must manually refine the mesh in these areas of stress concentrations. In a p-element code, the elements are unchanged but the order of the polynomial transfer function is iteratively increased up to order nine. This is done internally during the iterative solution process and does not require manual intervention. This type of system was well suited for the task of generating the experimental data since it was robust in handling large changes in the flexure geometry without manual intervention.

\section{Experiment Design and Analysis}

This section describes the construction and analysis of the experimental designs used to generate the mathematical models of the design space. Factorial experimental designs were used because they provide an efficient means to determine the influence and interaction among the design variables on the responses. Even though this is a computational experiment. the data is not without cost. Computational resources and manual manipulation of the results are required to obtain the data, and therefore the data volume should he minimized. Factorial experiment designs are well suited for this application.

In a classical one-factor-at-a-time approach, a single design variable would be varied while holding all other variables at a constant level. While this approach is intuitive to the designer, it is inefficient and more importantly could miss significant interactions among the design variables. With the OFAT approach, it is assumed that the effect of a single variable on the responses is the same regardless of the level of the other design variables. In real world situations, this is rarely the case. Factorial designs vary all of the variables simultaneously in a specified manner and enable the determination of the same main effects derived from an OFAT approach as well as significant interactions between the variables.

A two-level factorial design was used initially. These designs are useful in a broad array of applications but they are limited in one important respect: The fact that they feature only two levels of each design variable means that response models developed from data acquired with such designs can only accommodate first-order and mixed first-order terms. For example, in a two-variable, two-level factorial design with factors $\mathrm{A}$ and $\mathrm{B}$, it is possible to develop response models featuring the linear $A$ and $B$ terms and a second-order interaction term, $A B$. The pure quadratic terms $\left(\mathrm{A}^{2}\right.$ and $\left.\mathrm{B}^{2}\right)$ cannot be quantified. because this would require at least three levels of the design variables.

A useful extension to the two-level factorial design, accommodating full second-order response models, is the Box-Wilson. or Central Composite Design (CCD). A CCD for two variables is illustrated in figure 6 . The four data points comprising the square are the two-level factorial design points and are referred to as "corner points". They define all combinations of the high and low levels of the two variables. There are also a number of "center points" in this design that are 
replicates of the variable levels at the design center. Finally, there are four "axial points". also referred to as "star points", arranged on the axes of the variable coordinate system. A CCD features five levels of each design variable.

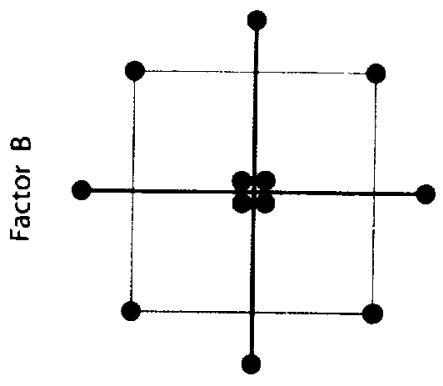

Factor A

Figure 6. Central Composite Design in two variables.

Although the description of the CCD has been given in a two-variable case, the design extends readily to any number of variables. While the details of the CCD are beyond the scope of this paper, many advantages flow from this design space geometry. The reader is referred to References 7,8 , and 9 for more details.

The first step in constructing the experimental design was to select the range of the design variables. The selection of the range proved to be a challenging aspect of this experiment. This was due to the need of choosing a wide enough range while simultaneously ensuring that the geometrical constraints were fulfilled. In general, to produce a useful model from an experimental design, the design variables must be varied over a large enough range to effect a meaningful change in the responses. For the initial design, the range was chosen to be as broad as possible. In subsequent designs, as we moved toward a response extrema. the range was narrowed.

Furthermore, the combination of design variables was constrained due to the physical geometry that they represent. An arbitrary combination of variable levels could violate the physical geometry. For example, the hole diameter must always be less than the overall width or height of the flexure. If the diameter were larger than one of these dimensions, it would cut through the sides of the flexure and thereby produce unacceptable geometry. Prior to executing the design, all of the combinations of the design variables were verified to ensure that the geometry was acceptable. If a particular combination was not acceptable then it was slightly modified instead of adjusting the range and generating a new design. Tailoring the design to meet the geometrical constraints proved to be tedious and somewhat limiting during the experimentation. The ability to mathematically define the physical constraints and build them into the experimental design is an arca of future research.

Three experimental designs were performed to determine a final flexure design. The first experiment involved the variables $A, D, E$, and $F$. These variables control the volume and location of the flexure. The goal of this first experiment was to define the center of the design in the search space. The design center is represented by a combination of variables that is near a combined response extrema. For this first design, the flexure was rectangular in shape. A four-variable full factorial design consisting of 16 runs was performed. The 16 geometries represented by this design are shown in figure 7 (the forward portion of the balance has been removed for clarity). Mathematical models consisting of the main effects and two-way interactions were derived. Using these models, the design center was approximated for these four variables.

The second design included the other six design variables that control the shape and contour of the flexure. Depending on the level of the design variables, the shape of the flexure was able to transition from a rectangle to a thin-walled octagonal cross section. A highly fractionated factorial design was executed to minimize computational resources. With 10 design variables, a full factorial would require 1024 runs. Not only is this an excessive number of runs, but also it is not necessary to derive an adequate model. A full factorial design in 10 variables allows for the resolution of all possible interactions including the main effects, two-way, three-way, and four-way, up to ten-way interactions. While it is possible that there are significant two-way and three-way interactions, it is unlikely that ten-way interactions would make a significant contribution to the model.

Therefore, a fractionated design enabled fewer experimental runs while giving up the capability to determine some multiple factor interactions. The consequence of a fractionated design is that some lower order terms will be aliased with higher order terms, thereby making it difficult to unambiguously resolve the lower order terms. A $1 / 64^{\text {th }}$ fraction of the full factorial was executed requiring only 16 combinations of the 10 design variables. The geometries represented by these 16 combinations are shown in figure 8 . The mathematical models derived from these design were then used to determine combinations of the design variables that would fulfill the response criteria. The analysis of this design revealed that it contained a combined response extrema. 


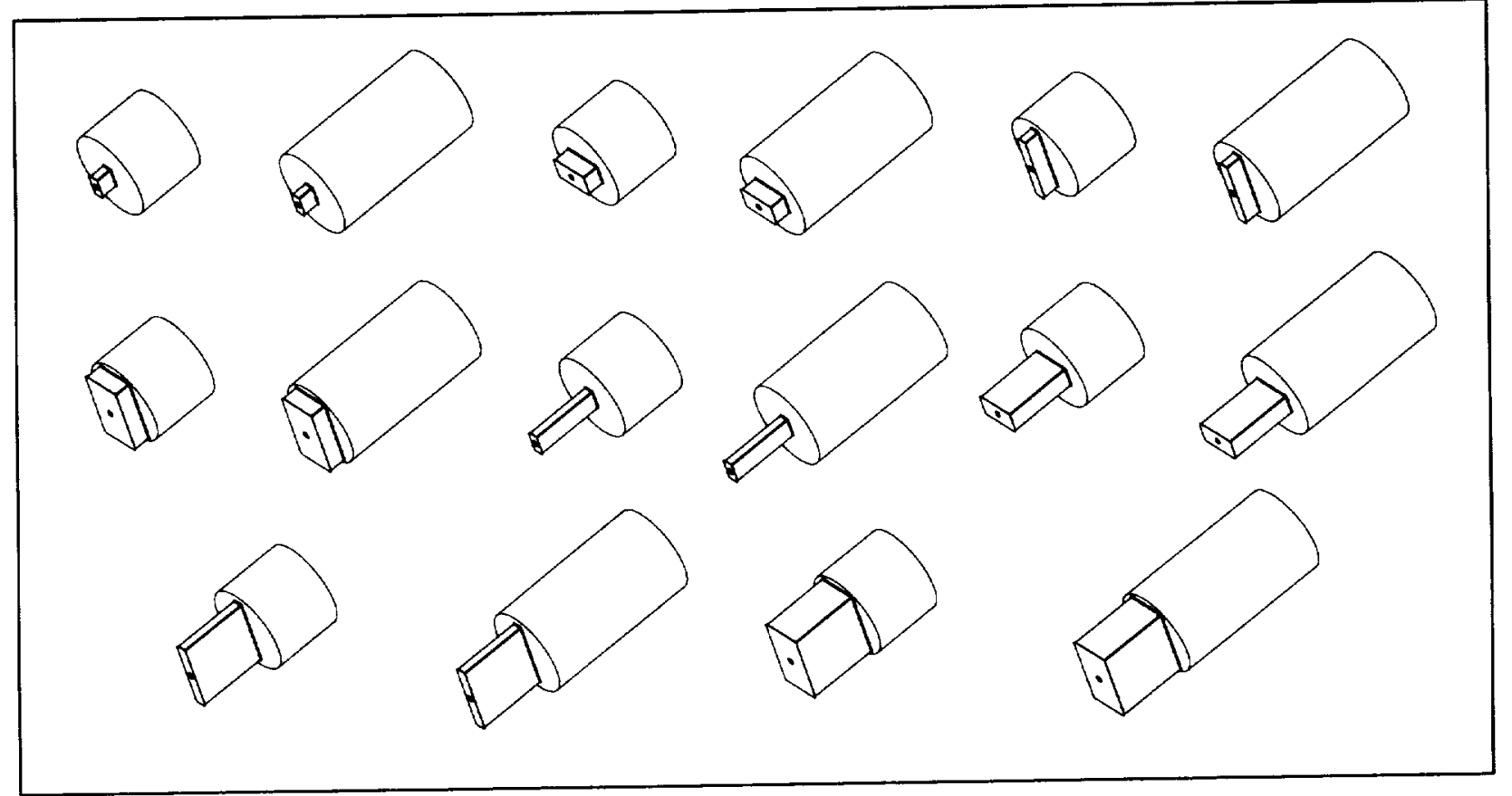

Figure 7. Four variable design geometries.

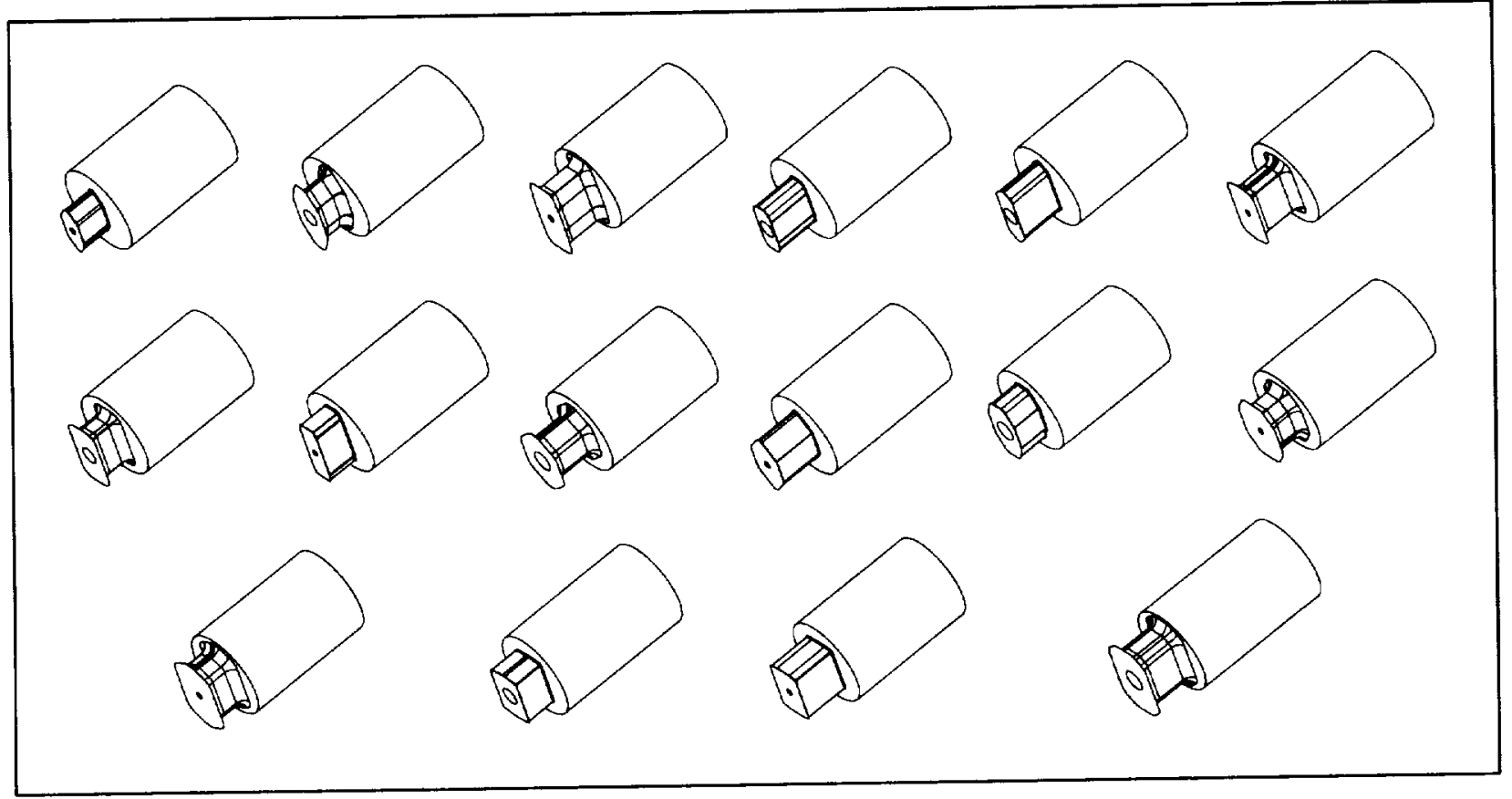

Figure 8. Ten variable fractional factorial design geometries. 
Therefore, in the third and final experiment. the fractionated factorial design in 10 variables was augmented to include axial and center points. This augmentation created a hybrid form of a central composite design. This design combined the 16 runs from the $1 / 64^{\text {th }}$ fraction with 20 axial points and 10 center points resulting in 46 total points. There were now five levels of each of the design variables. A single center point geometry was used and then simulated random errors were added to generate ten replicates. The inclusion of axial and center points enabled the determination of curvature in the math model. Therefore, the use of higher order terms increased the precision of the prediction and was important for the optimization of the variable levels. The geometries of the 21 additional points are shown in figure 9.
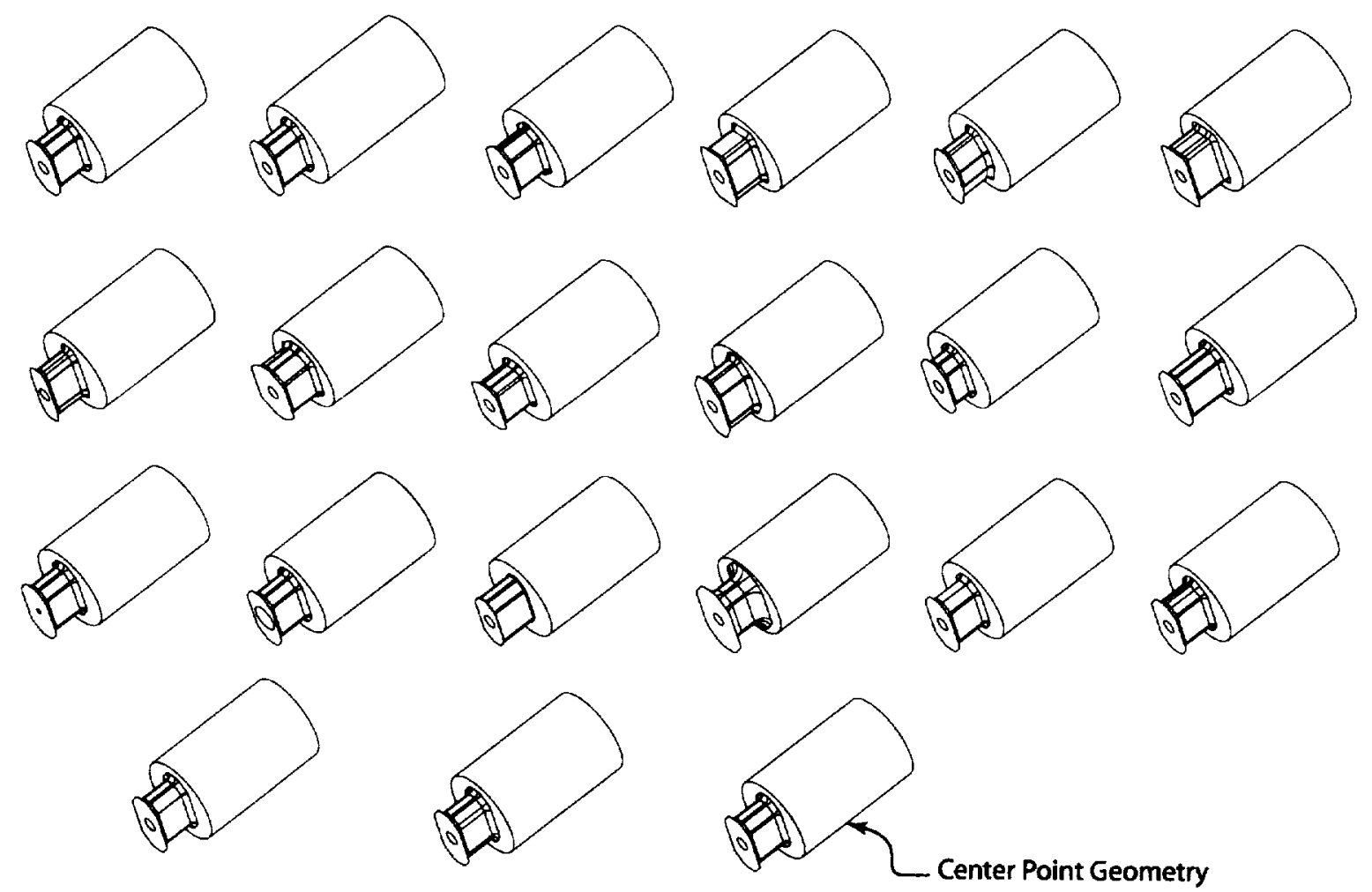

Figure 9. Augmented factorial design geometries.

\section{Desirability Function}

One complication in an optimization problem that features multiple response criteria is that the combination of independent variables that optimizes the design with respect to one response is not generally the combination that optimizes it for another. Derringer and Suich ${ }^{10}$ developed the concept of a "desirability function" to cope with such simations. The desirability function is a single quantitative response index ranging from 0 to 1 that approaches 1 more closely as various criteria specified by the designer are more closely met. Desirability functions are computed for each response individually. Calculating the geometric mean of all the individual functions then produces a single index. The desirability function can identify a solution that consists of some combination of independent variables that may not perfectly optimize the performance of the design with respect to any of the individual responses of interest, but which provides desirable performance for all of the responses.

For example, assume that it is unacceptable if a response level falls below some value, $\mathrm{A}$, and that the largest possible value between $\mathrm{A}$ and some goal value, $B$, is desired. Assume further that any response greater than or equal to B is equally acceptable. That is, we assume that the designer feels that a given response has to exceed the A level to be minimally acceptable and the larger the better after that, with all expectations met if the response level is $\mathbf{B}$ or more. The desirability function for this response variable is created by a transformation mapping the range from $A$ to $B$ into a 
range from 0 to 1. Response values less than $A$ are assigned a desirability value of 0 , and those greater than $B$ are assigned a value of 1 . If the desire is to achieve the largest possible value of the response, the value of " $B$ " can be set to the largest value of the response that can be achieved within the full range of independent variable settings.

Similarly, if response levels greater than some value, $B$, are unacceptable, and the smallest possible value between $\mathrm{B}$ and some minimal goal value. $\mathrm{A}$, is desired, and if any response as small or smaller than $A$ is equally acceptable, the desirability function is created by a transformation mapping the range from $A$ to $B$ into a range from 1 to 0 . Response values greater than $B$ are assigned a desirability value of 0 , and those less than $A$ are assigned a value of 1 . If the desire is to achieve the smallest possible value of the response, the value of "A" can be set to the smallest value of the response that can be achieved within the full range of independent variable settings.

It may simply be desirable for a particular response to lie with some range of values between $A$ and $B$. In that case, the desirability function is given a value of $I$ for responses between $\mathrm{A}$ and $\mathrm{B}$ and 0 for responses outside this range.

It is often desirable that a particular response lies as close to a specific target value as possible. In this case, the desirability function is designed to have a value of 1 at the target value and to approach zero as the response varies from the target in either direction, becoming zero when the response is unacceptably distant from the target.

It is not necessary for the desirability function of a specific response to vary linearly from 0 to 1 . A weighting constant, $w$, can be applied that imparts to the desirability function the designer's subjective evaluation of its role in the overall optimization. For example, the desirability function for a response to be maximized in the range of $A$ to $B$ can be constructed to have a value of 0 if the response is less than $A, I$ if the response is greater than $B$, and for responses between $A$ and $\mathrm{B}$, the desirability function can be described as follows:

$$
d_{l}=\left(\frac{\hat{y}_{1}-A}{B-A}\right)^{n}
$$

where $d_{i}$ is the desirability function for the $i^{t h}$ response, and $w$ is a weighting constant that is smaller when maximizing the response is relatively less important and larger when maximizing the response is relatively more important. Figure 10 shows how this function changes as the response varies between $A$ and $B$, for various values of $w$. Similar weightings can be applied for other objectives besides maximizing the response.

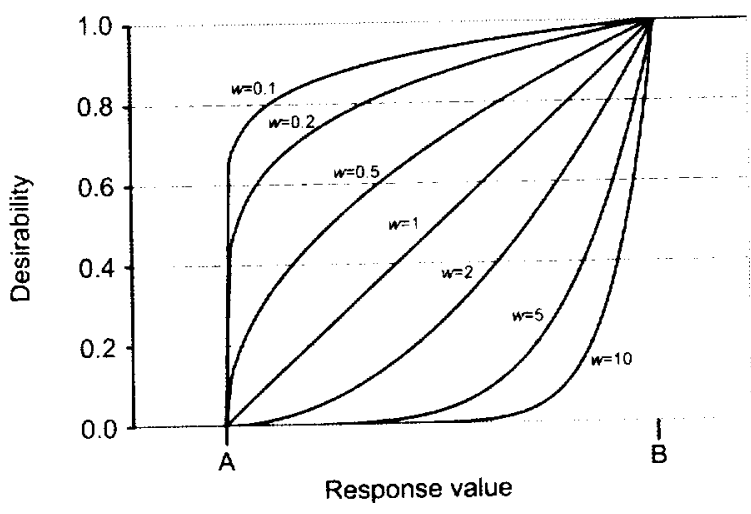

Figure 10. Desirability function for a response to be maximized.

After desirability values are computed for each response variable, they are combined into a single desirability index, $D$, by calculating their geometric mean. Further refinements to the weighting can be applied by assigning a range of numbers (from 1 to 5 , say) to the importance of optimizing each response variable. The final desirability index then is computed as follows:

$$
D=\left(\prod_{i=1}^{n} d_{i}^{v_{i}}\right)^{1 / \sum_{i}}
$$

where $v_{i}$ is a number indicating the relative importance of the $i^{\text {th }}$ response, which might typically be an integer in the range of 1 to 5 , with 5 indicating the greatest importance and 1 indicating the least.

In the present study, desirability functions were developed for the criteria that sensitivities for normal force, side force, yaw moment, and pitch moment all exceed 1000 microvolts per volt and be as large as possible, while the deflection of the balance in the $z$ and $y$ axis be minimized, and in any case less than 0.002 inches in the $z$ axis and 0.005 inches in the $y$ axis. The maximum stress intensity was specified to be less than 75.000 pounds per square inch, and as small as possible. Equal importance was assigned to all response criteria $\left(v_{i}=1\right.$ for all $\left.i\right)$ and linear weightings were applied ( $w=1.0$ for all responses). Combinations of the independent variables that maximized the grand desirability function, $D$, were chosen as the starting point for further refinements applied using graphical optimization, as outlined in the next section. 


\section{Graphical Optimization}

The desirability functions are based on the designer's subjective impression of the relative importance of various design objectives, and can best be thought of as a tool to achieve an approximately optimum single-point design. Further refinements on this initial design are possible using graphical optimization techniques that offer the designer additional insights into the tradeoffs among competing optimization criteria. These methods result in a range of independent variables throughout which all responses satisfy certain minimal criteria.

In this study, an initial flexure design was determined using desirability analysis as described above. Special computer software was then used to perform graphical optimization by exploiting the fact that extrema in the various response variables have associated with them closed contours of constant response value. Even if such an extremum lies beyond the range of independent variables considered, there will often be open contours that enclose some region of the design space bounded on one or more sides by a design space boundary.

It is possible to define contours for each response variable that correspond to some particular optimization criterion. For example, the contours can be defined that enclose all combinations of the independent variables for which a given response exceeds some minimum acceptable value or is less than some maximum acceptable value. Pairs of contours can be selected to bound combinations of independent variables for which the response is within some prescribed range. Overlaying such contours for different response criteria reveal at a glance whether there is a non-null intersection of all the criterion regions. If not, then it is possible to see which criteria require the least relaxation to achieve such an overlap. It is also possible to see that a relatively inconsequential relaxation of one or more criteria might dramatically increase the number of combinations of independent variables that satisfy all constraints. It could be quite beneficial to have a fairly wide range of independent variables that meet all response criteria. since certain combinations may be easier to implement than others or less expensive. Figure 11 is representative of a graphical optimization. The area highlighted in gray indicates combinations of the independent variables that satisfy all constraints. This region is known colloquially as a "sweet spot". and graphical optimization is therefore often described informally as "sweet spot analysis".

Once a region is defined by graphical optimization within which all response criteria are satisfied, a specific design point can be selected within this region to maximize or minimize some other factor of interest. For example, not all balance geometries that satisfy performance goals will be equally easy to manufacture. In such a case, the combination of geometric parameters that minimizes the manufacturing difficulties can be selected from within the region that satisfies all performance requirements. Developing such manufacturing cost functions is a next logical extension of the optimization procedures described in this paper.

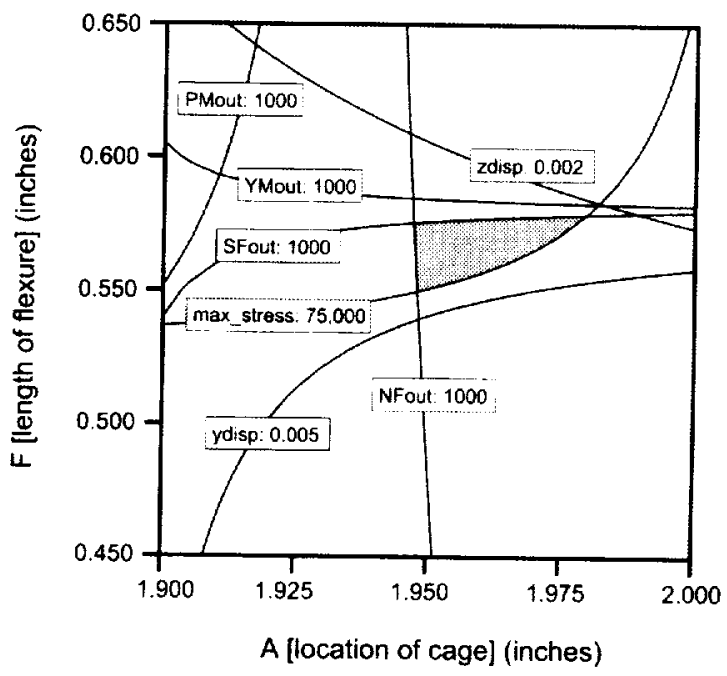

Figure 11. Graphical optimization.

In this case figure 11 illustrates design variable $\mathrm{F}$ (length of the flexure) plotted versus variable $A$ (location of the flexure). To overcome the limitation of not being able to plot all of the design variables simultaneously, which would require a ten dimensional plot, two are selected and all other variables are set to the constant levels. The contour lines represent the response criteria previously specified. The response boundaries that border the sweet spot are the normal force sensitivity, side force sensitivity, and the maximum stress intensity. All other response criteria are some distance from the boundaries of the sweet spot indicating that they are not limiting metrics for this particular combination of design variables. Any combination of variable $A$ and $F$ lying within the sweet spot will satisfy all of the specified criteria.

Based on this graphical optimization, the final flexure geometry was selected and is illustrated in figure 12 (only the flexure is shown for clarity). A combination of design variables in the upper left corner of the sweet spot was selected because that area represents the minimum stress intensity, while satisfying the sensitivity requirements. A finite element analysis was performed on the resulting geometry and 
was compared to the prediction of the mathematical models derived from the experimental design. The comparison is provided in Table III. All of the predicted values fall within the $99 \%$ prediction intervals of the model, except for the prediction of the displacement in the y-axis, which is slightly outside the interval. This level of agreement between the prediction and the actual FEA solution demonstrates the usefulness of the mathematical models for searching through the design space.

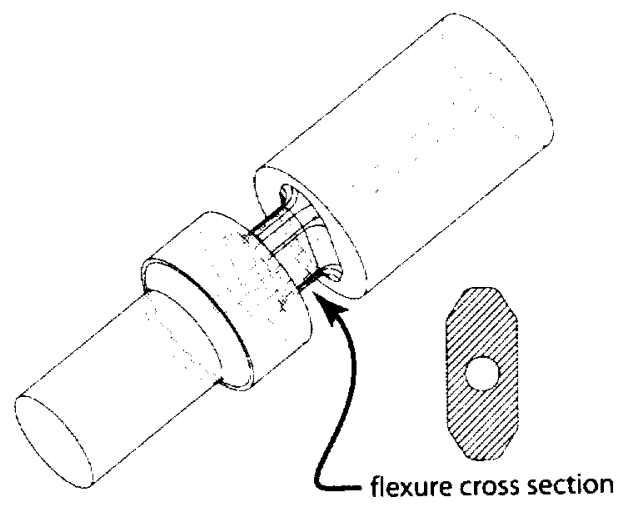

Figure 12. Final flexure geometry.

Table III. Predicted responses and FEA results.

\begin{tabular}{|l|r|r|}
\hline $\begin{array}{l}\text { Response } \\
\text { Variables }\end{array}$ & $\begin{array}{c}\text { Response Model } \\
\text { Prediction }\end{array}$ & FEA solution \\
\hline NF_out & $1004 \mu \mathrm{V} / \mathrm{V}$ & $982 \mu \mathrm{V} / \mathrm{V}$ \\
\hline PM_out & $1080 \mu \mathrm{V} / \mathrm{V}$ & $1052 \mu \mathrm{V} / \mathrm{V}$ \\
\hline YM_out & $1091 \mu \mathrm{V} / \mathrm{V}$ & $1152 \mu \mathrm{V} / \mathrm{V}$ \\
\hline SF_out & $1008 \mu \mathrm{V} / \mathrm{V}$ & $1073 \mu \mathrm{V} / \mathrm{V}$ \\
\hline zdisp & $1.96 \mathrm{E}-03$ inches & $1.99 \mathrm{E}-03$ inches \\
\hline ydisp & $3.33 \mathrm{E}-03$ inches & $3.86 \mathrm{E}-03$ inches \\
\hline max_stress & $73.457 \mathrm{psi}$ & $74,720 \mathrm{psi}$ \\
\hline
\end{tabular}

\section{Concluding Remarks}

A proposed method for performing a structural optimization of a force balance using a computational experiment design has been presented. An example optimization of a force halance measurement section has been illustrated demonstrating the feasibility of using this new method.

The powerful insights that are provided to the balance design engineer using graphical optimization analysis cannot be over emphasized. This graphical optimization tool enhances the intuition and experience of the designer regarding design tradeoffs and limiting boundaries created by the response criteria. Also, this type of analysis provides the balance designer with vital information regarding the complex, and often times subtle, underlying relationships between the design variables and the responses metrics.

There exists considerable interest in an automated balance design system. The proposed method is well suited for an automated system by relying on objective design decisions. In order to automate this method the experimental design and analysis software would need to be integrated with the solid modeling and finite element analysis software. While there are significant benefits to an automated system, the cost of software integration would need to be justified.

The proposed method can lead to the optimization of current geometrical structural designs and can also be used to generate advanced geometry. The technique can be extended to include other structural design requirements such as dynamic response, which would combine the response metrics of stiffness and mass. Non-traditional response metrics could also be considered including manufacturing time and cost. While this paper focused on a specific structural optimization application, similar response metrics are commonly found in a broad array of structural optimization applications.

\section{References}

1) Lindell, M.C.: Finite Element Analysis of a NASA Narional Transonic Facility Wind Tunnel Balance. First International Symposium on Strain Gauge Balances, October 1996.

2) Zhai, M.; Ewald, B.; and Hufnagel, K.: Shape Optimization of the Internal Tumnel Balances Using Simulated Biological Growth Approaches. AIAA-96-2255, $19^{\text {th }} \quad$ AIAA Advanced Measurement and Ground Testing Conference, June 1996

3) Zhai, M.: Optimization of the Performance of Internal Six-Component Strain-Gage Windtumel Balances with FEM. ICAS-96-2.4.3, 20 ${ }^{\text {th }}$ Congress on the International Council of the Aeronautical Sciences, September 1996.

4) Parker, P.A.: Cryogenic Balance Technology at the National Transonic Facility. AIAA-2001-0758. $39^{\text {th }}$ AIAA Aerospace Sciences Mecting and Exhibit, January 2001.

5) Parker, P.A.: Wind Tunnel Model System Dynamic Analysis and Simulations with Application to Model Sistem Vibration Suppression. Christopher Newport University, Department of Applied Physics and Computer Science, Thesis, 2000. 
6) Hou, J.W.: Twu, S.L.: Optimum Design of Internal Strain-Gage Balances: An Example of ThreeDimensional Shape Optimization. Journal of Mechanisms, Transmissions, and Automation in Design, June 1987.

7) Box, G. E. P.; Hunter, W. G.; and Hunter, J. S.: Statistics for Experimenters. An Introduction to Design. Data Analysis, and Model Building. New York: Wiley, 1978.

8) Montgomery, D. C.: Design and Analysis of Experiments, $5^{t_{1}}$ Ed. New York: Wiley, 2001.

9) DeLoach, R.: Improved Quality in Aerospace Testing Through the Modern Design of Experiments. AIAA-2000-0825. $38^{\text {th }}$ AIAA Aerospace Sciences Meeting and Exhibit, January 2000.

10) Derringer, G.: and Suich, R.: Simultaneous Optimization of Several Response Variables. Journal of Quality Technology. 12. 214-219, 1980. 

